\title{
Clasificación de estímulos visuales para control de drones
}

\author{
Eduardo Zecua, Irving Caballero, José Martínez-Carranza, Carlos A. Reyes \\ Instituto Nacional de Astrofísica Óptica y Electrónica, Puebla, \\ México \\ corichiedu@ccc.inaoep.mx, pirving01@ccc.inaoep.mx, \\ carranza@inaoep.mx, kargaxxi@inaoep.mx
}

\begin{abstract}
Resumen. Los experimentos con grabaciones Electro-encefalográficas (EEG) registran oscilaciones del potencial de membranas neurales. Estas señales representan un porcentaje elevado de la actividad cerebral tanto del pensamiento como del movimiento corporal. Por lo anterio, en este trabajo se explora el uso de EEG para la clasificación del movimiento ocular, detonado por la observación de algún estímulo visual,de tal forma que los movimientos reconocidos puedan ser utilizados para controlar un dron. Para esto se generó una base de datos que se divide en dos grupos: entrenamiento y prueba, generada de 5 sujetos con una media de edad de 28 años. Se procesó la señal tomando los valores estadisticos que representaran los cambios más significativos y generando los vectores característicos de cada una de las muestras. Una vez entrenada la red neuronal con estos parámetros, se procede a clasificar nuevas estancias y, dependiendo de la clasificación, se manda una instrucción o punto de ubicación a un dron controlado mediante un controlador proporcional integral derivativo (PID), el cual utiliza estimaciones de posición del dron obtenidas a través de un sistema de captura de movimiento VICON.
\end{abstract}

Palabras clave: EEG, FFT, redes neuronales, drones, PID.

\section{Visual Stimuli Classification to Control Drones}

\begin{abstract}
Experiments with Electro-encephalographic recordings (EEG) capture oscillations of the potential of neural membranes. These signals represent a high percentage of brain activity in both, thought and body movement. Therefore, in this study we explore the use of EEG for classification of eye movement, triggered by the observation of a visual stimuli, such that, the recognized movements can be used to control a drone. To achieve this, a database is generated and divided into two groups: training and testing, generated with five test subjects with an average age of 28 years. The signal was processed by taking the statistical values that represent the most significant changes and generating the characteristic vectors of each of the samples. Once the neural network was trained with these parameters, it proceeds to classify new instances and, depending on the classification, an instruction or location point is sent to a drone
\end{abstract}


controlled by an Proportional Integral Derivative controller (PID), which estimates the position of the drone through a motion capture Vicon system.

Keywords: EEG, FFT, neural networks, drones, PID.

\section{Introducción}

Los vehículos aéreos no tripulados (VANTs) o drones actualmente están siendo utilizados para distintos propósitos entre los cuales se encuentran la vigilancia, esfuerzos militares para proveer seguridad, reconocimiento táctico, entre otras [12. Por otra parte, los experimentos con grabaciones electroencefalográficas (EEG) están siendo utilizadas, cada vez más, como índices del proceso de trabajo de la memoria a lo largo de una variedad de tareas que involucran la operación de un VANT 14.

Las señales de EEG recolectadas en el cráneo humano son fluctuaciones de potenciales eléctricos que reflejan actividad en las estructuras cerebrales subyacentes, particularmente en la corteza cerebral debajo de la superficie del cuero cabelludo. Las oscilaciones que produce el EEG se clasifican en función de su relación con la estimulación y pueden ser espontáneas. Los datos que se observan sugieren una relación positiva entre los estímulos inducidos por estados estacionarios de potenciales evocados visualmente y de la actividad cerebral [1].

Cuando un estímulo sensorial corto se presenta, como una luz parpadeante o un movimiento del antebrazo de una persona,se produce una perturbación en las señales del EEG, la cual inicia después de un pequeño retraso del evento inicial (estímulo) y se esparce por alrededor de medio segundo o menos. Los cambios en la amplitud de la señal, debidos a la perturbación, son pequeños (a lo mucho, unos pocos micro-voltios), y no se aprecian a simple vista dentro de las líneas de actividad cerebral.

La gente puede responder únicamente a una pequeña cantidad de información sensorial presente en cualquier momento. Por ello, la selección de la información es necesaria para facilitar los problemas computacionales introducidos por el enorme número de señales presentes en las superficies sensoriales y para asegurarse que la gente responda a un estímulo que sea relevante a los objetivos de la investigación 3. Aunque muchos estudios han investigado la atención visual, la atención puede ser centrada en otros atributos (o dimensiones). La gente puede atender a, o "mirar hacia", cierta información visual específica (por ejemplo, un sombrero rojo que lleva un amigo entre una multitud). En este sentido, fijar la mirada en un atributo, mejora la precisión en la detección visual o discriminación de tareas [3].

Motivados por lo anterior, en este trabajo se describen resultados sobre el uso de EEG para llevar a cabo el control de un dron. Para esto, los EEG se utilizan para clasificar el movimiento ocular, el cual se genera a partir de la observación de estímulos visuales. Una vez que el movimiento ocular es reconocido, estos se 
traducen con comandos de control para ejectura 5 tareas: despegar; viajar hacia un punto A en el espacio; hacia un punto B; o hacia un punto C; y aterrizar. Los resultados obtenidos en este trabajo indican que los movimientos oculares se reconocen con un $71.2 \%$ de precision. Este porcentaje sin duda puede ser mejorado, no obstante, este porcentaje habla de la viabilidad de utilizar la metodología descrita en este trabajo, la cual involucra el uso de EEG para reconocer el movimiento ocular derivado de un estimulo visual y que puede integrarse en un sistema de control para drones.

Clasificar el movimiento ocular a partir de un estimulo visual es de interés en éste trabajo ya que sería deseable identificar ciertos tipos de moviento ocular, y no sólo eso, si no tambien gestos, muecas,o algún otra seña que permita detectar un posible estado de alerta del piloto mientras vuela el dron. Ésto último a raíz de posibles escenarios donde el piloto observa una situacion de peligro, pero no le da tiempo de usar el control remoto para controlar adecuadamente el vehículo. Sin embargo, si la situación de riesgo se considera como un estímulo visual que es observado por el piloto, dicha situación podría ser reconocido con un sistema basado en la metodología que se presenta en este trabajo, y por ende utilizar dicho reconocimiento para enviar un paro de emergencia al dron o algún comando que le permita alejarse del peligro.

De este modo y con el objetivo de describir con detalle el sistema propuesto, éste artículo se ha organizado de la siguiente manera: el trabajo relacionado se presenta en la sección 2; el sistema y sus componentes principales son descritos en la sección 3; los resultados son presentados y discutidos en la seccíon 4; y finalmente las conclusiones se desglozan en la sección 5 y agradecimientos en la sección 6 .

\section{Trabajos relacionados}

Científicos de la Universidad de Texas en San Antonio (UTSA) están tratando de convertir la ciencia ficción en realidad desarrollando la tecnología que permitirá a los soldados controlar remotamente un VANT únicamente con sus mentes. Seis profesores de diferentes de departamentos de la universidad trabajan en distintos proyectos que tienen que ver con el estudio de la interacción cerebro-máquina.

Pero UTSA no es la primera universidad con drones controlados con actividad cerebral. En 2013, el profesor Bin He de la Universidad de Minnesota fue la primera persona en mostrar el vuelo de un pequeño dron cuadrucóptero a través de un aro de globos, completamente controlado con una gorra con 64 sensores de electrodos colocada en la cabeza de una persona. Para volar el dron remótamente, el piloto imaginaba un puño. si se imaginaba un puño con la mano izquierda, el dron se desviaba a la izquierda. Las señales se enviaban de forma inalámbrica desde la computadora hacia el dron, para lo cual, primero decodificaba las señales cerebrales enviadas por la gorra y las retransmitía en forma de comandos que el dron debía seguir. 
La dinámica del EEG h sido utilizada para examinar los procesos cerebrales involucrados en tareas como detección visual de un objetivo [10] y rastreo visiomotora [8. Para el año 2010, se había alcanzado un concenso sobre la mejor aproximación para examinar los datos del EEG [9], pero, en particular, dos anchos de banda de frecuencia han recibido más atención. Se ha encontrado que la actividad en el rango alfa, frecuentemente disminuye con el incremento de la dificultad de la tarea, mientras que lo opuesto se ha observado en la actividad registrada en el rango teta, particularmente en los sitios de los electrodos de la línea media frontal[ $[5]$.

Esperimentos electrofisiológicos han mostrado que las neuronas en la corteza visual de los humanos sincronizan sus disparos a la frecuencia de luz parpadeante, originando respuestas en el EEG las cuales muestran la misma frecuencia que el estímulo parpadeante [13].

Varios autores han analizado la relación entre la frecuencia EEG y el desempeño de diferentes tareas. En general, a una frecuencia baja del EEG se le ha relacionado a una reacción más larga en el tiempo (Reaction Time) o a un mayor número de errores 15. La hipótesis más común fue que la relación de desempeño del EEG fue modulada por el nivel de alerta[11. Está fuera de toda duda que el nivel de alerta produce cambios tanto en el EEG como en el desempeño. Sin embargo, reportes previos de trabajos con niños han mostrado una correlación positiva entre la potencia delta del EEG en reposo, y el tiempo de respuesta (RT) y la relación de error en atención visual y tareas de memorización llevadas a cabo en diferentes sesiones.

\section{Descripción del sistema}

Para el desarrollo del sistema se emplearon varios equipos descritos en las secciones 3.1 y 3.2. Todos los procesos fueron realizados en una computadora con procesador Intel i7 de cuatro núcleos con $8 \mathrm{~Gb}$ de memoria RAM y sistema operativo Ubuntu 14.4 operando con el sistema operativo robótico (ROS). Un segundo equipo con un procesador AMD A10 con 12 Gb de RAM con sistema operativo Windows 10 y operando con Matlab 2015b. Todo el sistema se divide en dos partes: la primera se refiere a la adquisición de los datos del EEG incluyendo su clasificación y, la segunda etapa implica el control del dron utilizando un controlador PID para moverlo a la posición especificada dada la clasificación de las señales.

\subsection{Sistema de posicionamiento VICON}

El sistema de traqueo Vicon es un sistema de ubicación y seguimiento a partir de cámaras monoculares que, a través de luz infrarroja, envía un haz de luz a marcadores especiales con forma esférica y con un recubrimiento reflejante en el cual rebota la luz en todas direcciones hacia las cámaras. Dependiendo de la cantidad de luz reflejada y la ubicación de cada uno de los marcadores, cada cámara hace una triangulación regresando la ubicación de cada marcador 
en el espacio con respecto a una referencia propuesta durante la calibración del equipo y con precisión milimétrica. Estos marcadores se colocaron en el cuerpo del dron de manera que no estorbaran en el vuelo y pudieran ser localizados en todo momento por el sistema de traqueo.

\subsection{Dron BEBOP}

Para el desarrollo de este proyecto se utilizó el dron BEBOP de la marca PARROT, el cual cuenta con una cámara monocular de tipo ojo de pescado y 4 hélices. Éste es controlado mediante WiFi a través de computadoras o celulares y cuenta con un sensor ultrasónico ubicado en parte inferior que mide la distancia entre él y el suelo lo que permite que se mantenga en vuelo de manera estable. Este modelo cuenta con protecciones para poder operarlo en interiores y exteriores (1).

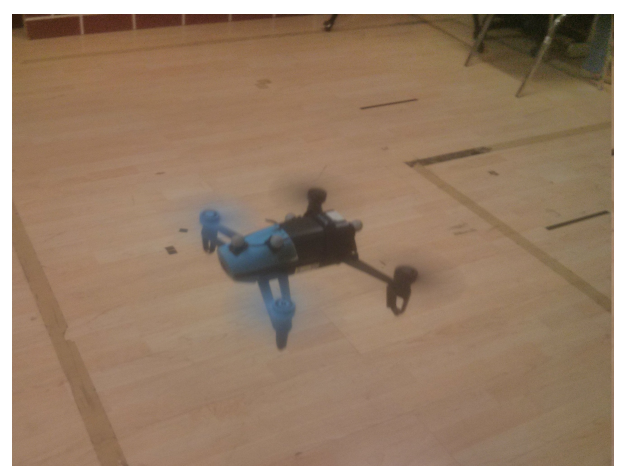

Fig. 1. BEBOP operando en vuelo

\subsection{Control PID}

Un controlador PID (Proportional Integral Derivativo) es un mecanismo de control genérico sobre una retrolimentación de bucle cerrado, ampliamente usado en la industria para el control de sistemas. El PID es un sistema que recibe un error calculado a partir de la salida deseada menos la salida obtenida; y su salida es utilizada como entrada en el sistema que queremos controlar. El controlador intenta minimizar el error ajustando la entrada del sistema.

El controlador PID consiste de tres parámetros distintos: el proporcional, el integral, y el derivativo. El valor Proporcional depende del error actual. El Integral depende de los errores pasados y el Derivativo es una predicción de los errores futuros. La suma de estas tres acciones es usada para ajustar al proceso por medio de un elemento de control como la posición de una válvula de control o la potencia suministrada a un calentador. 
Cuando no se tiene conocimiento del proceso, históricamente se ha considerado que el controlador PID es el controlador más adecuado. Ajustando estas tres variables en el controlador PID, puede proveer una acción de control diseñado para los requerimientos del proceso en específico. La respuesta del controlador puede describirse en términos de la respuesta del control ante un error, el grado el cual el controlador sobrepasa el punto de ajuste, y el grado de oscilación del sistema. Nótese que el uso del PID para control no garantiza control óptimo del sistema o la estabilidad del mismo.

Para el sistema de control del Dron se utiliza un PID que calcula el error entre el valor medido y el valor deseado. Esto se aplicó cuando se le asignaba un punto al dron al que tuviera que acercarse, midiendo con el sistema Vicon la posición y calculando el error (distancia entre la posición actual y la deseada). El PID ajusta los valores para acercarse al punto lo más rápido posible con un amortiguamiento en la disminución de la velocidad del vehículo conforme se va acercando al punto deseado. El método fue implementado para el control del desplazamiento del dron y para el giro en su propio eje.

Este modelo se divide en 3 partes:

- La parte proporcional, ajusta la velocidad dependiendo de la distancia entre el punto, esto se refiere a que mientras la distancia entre la posición actual y la deseada sea grande la velocidad del vehículo también será grande y viceversa.

- La parte integral va acumulando el error, esto se refiere a que mientras más tiempo tarde el vehículo en llegar al área deseada, éste acumula los valores para ir incrementado la velocidad en cierta medida.

- La parte derivativa mide la diferencia del error actual y error pasado, mide la proporción de cambio en cada uno de los instantes, lo cual ayuda al sistema a acelerar desde el principio puesto que es cuando el error es mas grande.

El ajuste de las ganancias de estos sistemas no es trivial y requiere ser modificado dependiendo de las condiciones de cada sistema, así como las condiciones de su entorno, ya que el dron es perturbado por el moviendo de sus propias hélices en vuelo.

\subsection{Adquisición de señales EEG}

Las frecuencias del EEG tradicionalmente se han clasificado en diferentes bandas. La actividad Delta $(1.5-3.5 \mathrm{~Hz})$ es la principal característica del sueño, pero puede estar presente durante la atención a procesos internos como cálculos mentales y memorización [6. La actividad Teta $(4-7 \mathrm{~Hz})$ puede reflejar una función portera del flujo de información a través del hipocampo y los circuitos de estructuras objetivo. La actividad Alfa $(8-13 \mathrm{~Hz})$ es más que una frecuencia espontánea y es un prototipo de procesos dinámicos que gobiernan un gran conjunto de funciones cerebrales integrativas. Los patrones Alfa puden ser espontáneos, inducidos o evocados por estímulos, movimientos o relaciondos a la memoria. La cuarta actividad es la Gama $(25-100 \mathrm{~Hz})$ la cual se ha teorizado que podrían estar implicadas en el proceso de percepción consciente [2]. 
Para la adquisición de las señales se utilizó la diadema de la marca Emotiv modelo EPOC, la cual consta de 14 canales distribuidos en la corteza craneal como se muestra en la figura (2). El sistema tiene una frecuencia de muestro de 128 SPS y se conecta a una computadora de manera inalámbrica a través de una conexión USB. El software utilizado para guardar todas las pruebas realizadas es el Emotiv TestBench de la marca Emotiv.

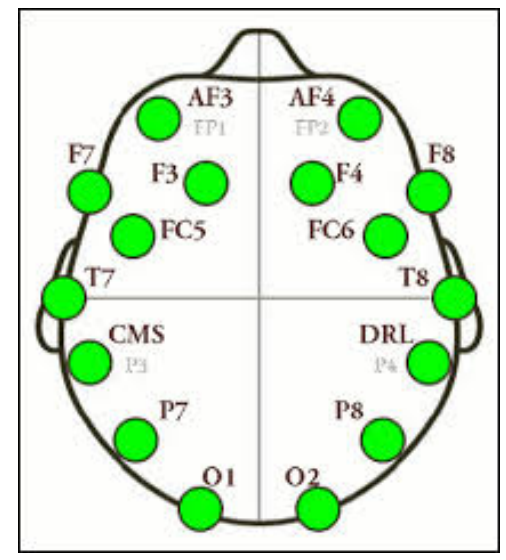

Fig. 2. BEBOP operando en vuelo

La base de datos se creó con cinco sujetos con una media de edad de 28 años. Las muestras se recopilaron mientras ellos estaban sentados y mirando una pantalla con una cruz roja en el centro y fondo blanco para que éstas tuvieran la menor cantidad de ruido al mover los ojos por distraccion de manera involuntaria como se aprecia en la figura (3). A los sujetos se les pidió que realizaran un movimiento ocular determinado (mirar hacia arriba, mirar hacia abajo, mirar a la izquierda, mirar a la derecha, parpadear) y cada vez que llevaban a cabo el movimiento, éste se marcaba con una etiqueta diferente en las lecturas del EEG.

\subsection{Procesamiento de la señal}

Una vez adquiridas las señales estas se guardaban con la extensión. EDF, para poder leer los datos y poder procesarlos se creó un programa en MATLAB que leyera cada una de las filas y descompusiera este formato en una matriz con los valores de cada uno de los 14 canales por separado.

De la matriz obtenida se seleccionan los canales más característicos para el movimiento ocular, estos se encuentran en la parte frontal arriba de los ojos con la diadema EMOTIV tenemos 4 canales (AF3, AF4, F7, F8) [4 que se encuentran cercanas a esta área. De estos cuatro canales se obtienen los valores estadisticos correspondinetes a los valores maximos y minimos que componen 


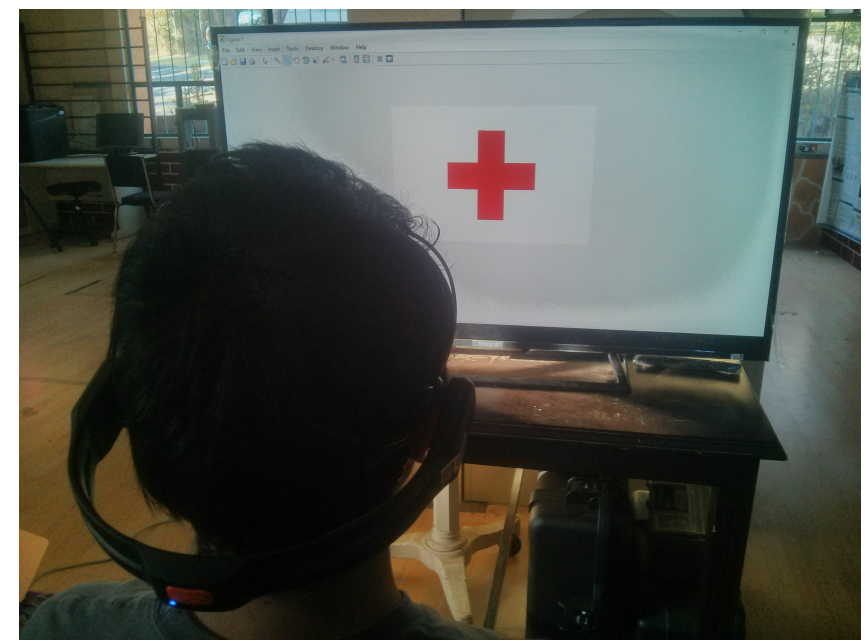

Fig. 3. Montaje de display con cruz roja en el centro

a la señal en el instante en que se generó el movimiento ocular, estos tiene variaciones dependiendo de como se genera el movimiento [7.

\subsection{Entrenamiento de la red neuronal}

Las pruebas del experimento se dividieron en dos partes, la primera consistía en recopilar muestras para el entrenamiento de una red neuronal creada con ayuda del toolbox de Matlab, siendo una red neuronal de tipo feed-fordward con 5 neuronas en la capa entrada, 8 neuronas en la capa de oculta y 5 neuronas en la capa de salida. Se usó el algoritmo de aprendizaje trainbr el cual se utiliza para ajustar los pesos en la fase de entrenamiento, consistiendo en un proceso de regularización bayesiana y fijando en 500 el número de épocas de entrenamiento, con el fin de ajustar los pesos de la red de manera óptima para la clasificación.

Es importante hacer mención que los datos de entrada se etiquetaban con cada uno de los movimientos oculares, proceso que se llevó a cabo mediante un programa generado en Matlab que enviaba los marcadores a través de un clic del ratón para especificar el instante en el que se realizó la acción.

En la segunda parte, se procesaron las muestras para la etapa de evaluación pasándolas por la red neuronal entrenada sin etiquetas, con el objetivo de cuantificar el porcentaje en que las nuevas instancias eran clasificadas correctamente.

\subsection{Sistema ROS}

Para el control todo el sistema se utilizó el sistema ROS, este es un software especializado en el control de robots el cual funciona con nodos que son lanzados desde códigos en $\mathrm{C}++$ o Python, con la ventaja de poder publicar en cada suscribirse a cada uno de ellos. Este sistema se utilizó para controlar el dron, 
el modelo que se utilizó para este experimento fue el BEBOP de la marca PARROT, este se conecta mediante wifi a una computadora o teléfono, para el control se creó un programa que se suscribiera al nodo de clasificación, una vez que este nodo publicara algún mensaje, dependiendo de la clasificación se toma la acción de despegar, aterrizar o volar el dron a una determinada área. La retroalimentación del dron se realizó el área donde este opera con el equipo VICON para saber su posición y la distancia de cada uno de los 3 puntos marcados.

\section{Resultados y experimentos}

La evaluación del clasificador se llevó a cabo con la base de datos de prueba, este conjunto contenía nuevas instancias que no había visto el clasificador. Una vez pasando por la red neuronal, ésta las clasificaba conforme había aprendido en la fase de enteramiento. Se calculó un arreglo de 5 números formados en columnas como se muestra en el Cuadro 1, cada uno de estos números corresponde al peso de la medida a la instancia que puede pertenecer cada vector. Para hacer la elección de la clase a partir de estos valores, se toma el número mayor y la clase final está dada por la posición de este número, por ejemplo, se puede observar en la columna P1 que el número mayor corresponde a la clase 1. Para construir la tabla se tomaron 10 muestras para poder observar cómo opera el sistema. Estos valores se tomaron al azar de la datos de prueba que cosiste en la combinación de las muestras de los sujetos.

Una vez que se tienen los resultados de pasar todas las muestras de prueba por la red neuronal para ser clasificados, éstas se comparan con los valores de la clase a la que realmente pertenecen. Dichos resultados se presentan en la Matriz de Confusión mostrada en la tabla (2). Cada fila representa la clasificación hecha por la red y las columnas representa la clasificación correcta. En la última fila y columna se muestran los porcentajes de las clasificaciones correctas hechas por el sistema, teniendo el total de aciertos en la celda de la última fila y columna. La posición $(1,1)$ de la tabla nos indica el número de elementos que clasificó correctamente el sistema de la clase 1 , en la posición $(1,2)$ nos indica cuantos elementos de la clase 1 fueron clasificados como la clase 2 y así sucesivamente.

Tabla 1. Resultados obtenidos de la clasificación de 10 estimulos. El valor más alto corresponde a la clasificación realizada por el clasificador

\begin{tabular}{|l|c|c|c|c|c|c|c|c|c|c|}
\hline Clasificador & P.1 & P.2 & P.3 & P.4 & P.5 & P.6 & P.7 & P.8 & P.9 & P.10 \\
\hline Clase 1 & 0.8111 & 0.7856 & 0.1636 & 0.1058 & 0.0008 & 0.2229 & 0.5004 & 0.0359 & 0.5828 & 0.2852 \\
\hline Clase 2 & -0.5137 & 0.0866 & 0.5636 & -0.0775 & -0.1450 & 0.1178 & 0.0919 & -0.1162 & -0.5676 & 0.4474 \\
\hline Clase 3 & -0.1255 & 0.1834 & -0.0006 & 0.9726 & 0.0090 & -0.1550 & 0.1632 & 0.0138 & 0.2187 & 0.1632 \\
\hline Clase 4 & 0.7191 & 0.0412 & -0.0136 & -0.1768 & 1.1190 & -0.0426 & 0.1715 & 1.0535 & -0.0550 & -0.0060 \\
\hline Clase 5 & 0.1196 & -0.0991 & 0.2896 & 0.1682 & 0.0215 & 0.8565 & 0.0750 & 0.0175 & 0.8018 & 0.1107 \\
\hline
\end{tabular}


Eduardo Zecua, Irving Caballero, José Martínez-Carranza, Carlos A. Reyes

Tabla 2. Matriz de confusión

\begin{tabular}{|l|c|c|c|c|c|c|}
\hline Clases & Clase 1 & Clase 2 & Clase 3 & Clase 4 & Clase 5 & \\
\hline Clase 1 & 29 & 1 & 1 & 2 & 5 & 67.9 \\
\hline Clase 2 & 5 & 17 & 0 & 0 & 8 & 56.7 \\
\hline Clase 3 & 3 & 0 & 16 & 0 & 4 & 69.6 \\
\hline Clase 4 & 1 & 0 & 0 & 28 & 0 & 96.6 \\
\hline Clase 5 & 3 & 4 & 3 & 0 & 19 & 65.5 \\
\hline & 66.7 & 77.3 & 80.0 & 93.3 & 52.8 & 71.2 \\
\hline
\end{tabular}

En los 10 casos de prueba mostrados en el cuadro 1, la prueba 2 (P.2) y la prueba 7 (P.7) fueron erróneas ya que las clases calculadas fueron "1. ${ }^{\mathrm{en}}$ ambas,

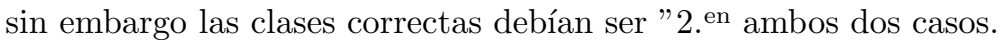

Para el control del vehículo las instrucciones se mandaron mediante un arreglo de 3 números, el primer digito contenía la instrucción de despegar, este se obtuvo de la clasificación 2 (mirar hacia arriba); el segundo digito especificaba a cuál de los 3 puntos pre-establecidos tenía que llegar, obtenido de las clasificaciones 3,4,5 (mirar a la izquierda, mirar a la derecha o parpadear); el ultimo digito contenía la instrucción de aterrizar, obtenido de la clasificación 1 (mirar hacia abajo); estas posibles acciones se describen en el Cuadro (3). Estos se escogieron de esta manera por lo inusual de su comportamiento en un estado normal. Debido a que es más factible parpadear, esta acción se tomó como un valor intermedio para ir a un punto en el espacio. Para que el sistema pudiera empezar a operar es necesario que el sujeto mire hacia arriba para poder despegar el dron. El sistema está diseñado para leer los tres parámetros antes mencionados en este orden. Si recibiera cualquier otro estimulo antes de despegar este no lo tomaría en cuenta. Una vez llevada a cabo la clasificación de los movimientos oculares y poder ejecutar el control del dron, estos valores son impresos en un archivo de texto (.TXT) para, posteriormente, ser leídos por un nodo de ROS y, dependiendo de la clase escrita, el dron realice las acciones predeterminadas. Para las pruebas se realizaron 10 experimentos los cuales se muestran el Cuadro (3).

Tabla 3. Clasificación de 10 experimentos realizados. La primer fila indica el número del experimento. La segunda fila se refiere a la primera acción (despegar). La tercera fila es la acción de movimiento a un punto definido mediante el control PID. La cuarta fila es la acción de aterrizar

\begin{tabular}{|c|c|c|c|c|c|c|c|c|c|}
\hline 1 & 2 & 3 & 4 & 5 & 6 & 7 & 8 & 9 & 10 \\
\hline $2-2$ & $2-2$ & $2-2$ & $2-2$ & $2-2$ & $2-2$ & $2-2$ & $2-2$ & $1-2$ & $2-2$ \\
\hline $4-4$ & $3-3$ & $4-4$ & $1-5$ & $5-5$ & $5-5$ & $4-4$ & $5-5$ & $4-4$ & $4-4$ \\
\hline $1-1$ & $1-1$ & $1-1$ & $1-1$ & $1-1$ & $4-1$ & $1-1$ & $1-1$ & $4-1$ & $1-1$ \\
\hline
\end{tabular}

El cuadro (3) se divide en columnas las cuales separan cada uno de los experimentos. Cada una de las filas (de arriba hacia abajo) representan los valores ordenados que tomaron en cada uno de los instantes, cada una de estas contiene 
dos números, el primero indica el valor que fue capturado en el clasificador y el segundo es el valor de la clasificación real, este último se anexa a la tabla (4). para comprar cada una de las instrucciones.

Tabla 4. Acciones llevadas a cabo de acuerdo a la clasificación de estímulos

\begin{tabular}{|c|c|c|}
\hline Clase & Estímulo & Acción \\
\hline Clase 1 & Mirar abajo & Aterrizar \\
\hline Clase 2 & Mirar arriba & Despegar \\
\hline Clase 3 & Mirar derecha & Mover a punto 1 \\
\hline Clase 4 & Mirar izquierda & Mover a punto 2 \\
\hline Clase 5 & Parpadeo & Mover a punto 3 \\
\hline
\end{tabular}

\section{Conclusiones y trabajo futuro}

En este trabajo se han presentado resultados de un sistema basado en el uso de EEG para el reconocimiento de movimiento ocular detonado por un estímulo visual y que, al reconocerse, se utiliza para enviar comandos de control a un dron. Las oscilaciones que produce el EEG pueden ser clasificadas en función de su relación con la estimulación. En este caso, los estímulos correspondieron al movimiento ocular de los sujetos de prueba y dichas oscilaciones se clasificaron en cinco clases distintas con las cuales se pudo accionar un dron con cinco diferentes acciones, dependiendo de la clase enviada. El clasificador obtenido tiene una precisión del $71.2 \%$, lo cual fue suficiente para los propositos de este trabajo y en medida de que el dron llevo a cabo las acciones determinadas sin mayor dificultad.

No obstante, para el trabajo a futuro se tiene considerado el mejorar la clasificación y complementarla con un detector de falsos positivos pues enviar un comando erroneo al dron puede ser catastrófico. Tambien se considera trabajar con el reconocimiento de algun otro tipo de expresión tales como muecas, pestañeos e incluso pensamientos. Del mismo modo, se contempla experimentar con diferentes estímulos visuales que generen una reacción de alerta en el piloto tales como el observar que el dron se encuentre en riesgo de chocar. Finalmente, también se realizaran pruebas en escenarios menos controlados, como por ejemplo, realizar la detección y el control del dron en ambientes exteriores.

Agradecimientos. Este trabajo fue financiado por la Royal Society-Newton Advanced Fellowship con referencia NA140454. El autor Eduardo Zecua Corichi agradece el apoyo recibido por parte de CONACYT bajo la beca número 624062 . El autor Irving Caballero Ledesma agradece el apoyo recibido por parte de CONACYT bajo la beca número 702771. 


\section{Referencias}

1. Başar-Eroglu, C., Strüber, D., Schürmann, M., Stadler, M., Başar, E.: Gammaband responses in the brain: a short review of psychophysiological correlates and functional significance. International Journal of Psychophysiology 24(1), 101-112 (1996)

2. Buzsaki, G.: Rhythms of the Brain. Oxford University Press (2006)

3. Corbetta, M., Miezin, F.M., Dobmeyer, S., Shulman, G.L., Petersen, S.E.: Attentional modulation of neural processing of shape, color, and velocity in humans. Science 248(4962), 1556 (1990)

4. Croft, R., Barry, R.: Removal of ocular artifact from the eeg: a review. Neurophysiologie Clinique/Clinical Neurophysiology 30(1), 5-19 (2000)

5. Gevins, A., Smith, M.E., McEvoy, L., Yu, D.: High-resolution eeg mapping of cortical activation related to working memory: effects of task difficulty, type of processing, and practice. Cerebral cortex 7(4), 374-385 (1997)

6. Harmony, T., Fernández, T., Silva, J., Bernal, J., Díaz-Comas, L., Reyes, A., Marosi, E., Rodríguez, M., Rodríguez, M.: Eeg delta activity: an indicator of attention to internal processing during performance of mental tasks. International journal of psychophysiology 24(1), 161-171 (1996)

7. Herrmann, C.S.: Human eeg responses to 1-100 hz flicker: resonance phenomena in visual cortex and their potential correlation to cognitive phenomena. Experimental brain research 137(3-4), 346-353 (2001)

8. Huang, R.S., Jung, T.P., Delorme, A., Makeig, S.: Tonic and phasic electroencephalographic dynamics during continuous compensatory tracking. NeuroImage 39(4), 1896-1909 (2008)

9. Klimesch, W., Freunberger, R., Sauseng, P., Gruber, W.: A short review of slow phase synchronization and memory: evidence for control processes in different memory systems? Brain research 1235, 31-44 (2008)

10. Makeig, S., Delorme, A., Westerfield, M., Jung, T.P., Townsend, J., Courchesne, E., Sejnowski, T.J.: Electroencephalographic brain dynamics following manually responded visual targets. PLoS Biol 2(6), e176 (2004)

11. Makeig, S., Jung, T.P.: Tonic, phasic, and transient eeg correlates of auditory awareness in drowsiness. Cognitive Brain Research 4(1), 15-25 (1996)

12. Parasuraman, R., Cosenzo, K.A., De Visser, E.: Adaptive automation for human supervision of multiple uninhabited vehicles: Effects on change detection, situation awareness, and mental workload. Military Psychology 21(2), 270 (2009)

13. Picton, T.: Human brain electrophysiology. evoked potentials and evoked magnetic fields in science and medicine. Journal of Clinical Neurophysiology 7(3), 450-451 (1990)

14. Roberts, D.M., Taylor, B.A., Barrow, J.H., Robertson, G., Buzzell, G., Sibley, C., Cole, A., Coyne, J.T., Baldwin, C.L.: Eeg spectral analysis of workload for a part-task uav simulation. In: Proceedings of the Human Factors and Ergonomics Society Annual Meeting. vol. 54, pp. 200-204. SAGE Publications (2010)

15. Valentino, D.A., Arruda, J., Gold, S.: Comparison of qeeg and response accuracy in good vs poorer performers during a vigilance task. International Journal of Psychophysiology 15(2), 123-133 (1993) 